\title{
Disorder-immune confinement of light in photonic-crystal cavities
}

\author{
Alejandro Rodriguez, M. Ibanescu, J. D. Joannopoulos, and Steven G. Johnson \\ Center for Materials Science and Engineering, Massachusetts Institute of Technology, \\ Cambridge, Massachusetts 02139
}

\begin{abstract}
Received July 1, 2005; revised manuscript received August 5, 2005; accepted August 11, 2005
We demonstrate by finite-difference time-domain simulations in 2D and 3D that optical cavities in realistic finite photonic crystals have lifetimes and modal volumes that are essentially insensitive to disorder (of various types, including surface disorder and randomized positions), even with unphysically large disorder. A lifetime $Q=10^{8}$ is demonstrated in a 3D single-mode cavity with a half-wavelength mode diameter using only eight vertical periods of a disordered crystal. (C) 2005 Optical Society of America

OCIS codes: $230.3990,230.5750,240.5770$.
\end{abstract}

In this Letter, we show that a photonic-crystal cavity can confine light with a lifetime that is not limited by fabrication disorder and is independent of modal volume. A photonic crystal is a periodic dielectric structure with a photonic bandgap, a range of wavelengths in which light cannot propagate in the crystal; a point defect introduced into such a crystal traps a localized mode. ${ }^{1}$ Dielectric cavities can also be made by ring resonators ${ }^{2}$ and by point defects in photonic-crystal slabs that combine bandgaps in one or two directions with index guiding ${ }^{3}$; all such cavities, however, suffer from intrinsic radiation losses whose reduction ultimately requires a trade-off in modal volume ${ }^{3-6}$ (except for Ref. 7), and any disorder should also scatter light and limit the lifetime. With a complete photonic bandgap (i.e., for all directions and, in 3D, all polarizations), the dimensionless lifetime $Q$ of the cavity increases exponentially with the number of crystal periods surrounding the cavity, independent of its modal volume (which can approach a half-wavelength diameter). ${ }^{1}$ Moreover, it has been shown that the photonic bandgap is preserved even when a small (bounded) amount of disorder is introduced into the crystal, ${ }^{8-11}$ leading to the hope that optical confinement is maintained in a disordered cavity (with some evidence in 2D provided by Ref. 12). Here we show that this hope is justified: by finitedifference time-domain ${ }^{13}$ (FDTD) simulations for crystals in both $2 \mathrm{D}$ and $3 \mathrm{D}$, we demonstrate that the only effect of the disorder is a slight change in the exponential rate at which $Q$ increases with the number of crystal periods, and also a small shift in the cavity frequency $\omega$ that could be compensated for by introducing a single external tuning parameter into the cavity. ${ }^{14-17}$ We obtain a $Q$ of $10^{8}$ optical periods and a half-wavelength single-mode diameter in a small (finite) 3D crystal with unphysically large fabrication disorder.

Thus, with a complete-gap crystal, the only factors limiting the lifetime of a cavity are the size of the crystal that can be fabricated and the intrinsic absorption losses of the materials. For example, $\mathrm{Si}$ at $\lambda=1.55 \mu \mathrm{m}$ has a bulk extinction coefficient $k$ that is so small that it is difficult to measure, but an extrapolation of the band-edge $k$ tail $^{18}$ is $\sim 10^{-12}$, giving a maximum $Q>10^{12}$; before this point, however, electronic surface states and similar effects determined by fabrication details may dominate absorption. The resulting high $Q$ and small modal volume are important especially for nonlinear devices to minimize the power requirements. ${ }^{19}$ Note that, although $1 / Q$ is a fractional bandwidth, one actually wants $Q$ to be much larger than a design bandwidth might suggest, since the $Q$ here is only that of radiative loss: the ratio of twice the total $Q(\sim \omega /$ bandwidth $)$ to the loss $Q$ is the fractional loss. ${ }^{20}$ For comparison, we point out that past dielectric cavities with very high $Q$ values required a substantial sacrifice in modal volume compared to the theoretical optimum $\approx(\lambda / 2 n)^{3}$, such as microspheres $\left(Q \approx 10^{10}\right.$, modal volume $>10^{4}$ $\times$ optimum $^{21}$ or photonic-crystal heterostructures $\left(Q \approx 10^{6} \text {, modal volume }>10 \times \text { optimum }\right)^{6}$

We focus on two structures: in 2D, a square lattice of dielectric rods in air (period $a$, radius $0.2 a, \epsilon=12$ $\sim \mathrm{Si}$ ), shown in the inset of Fig. 1, which has a gap (frequencies $0.286-0.4212 \pi c / a$ ) for the TM polarization (E out of the plane) ${ }^{1}$; in $3 \mathrm{D}$, an fcc lattice (lattice

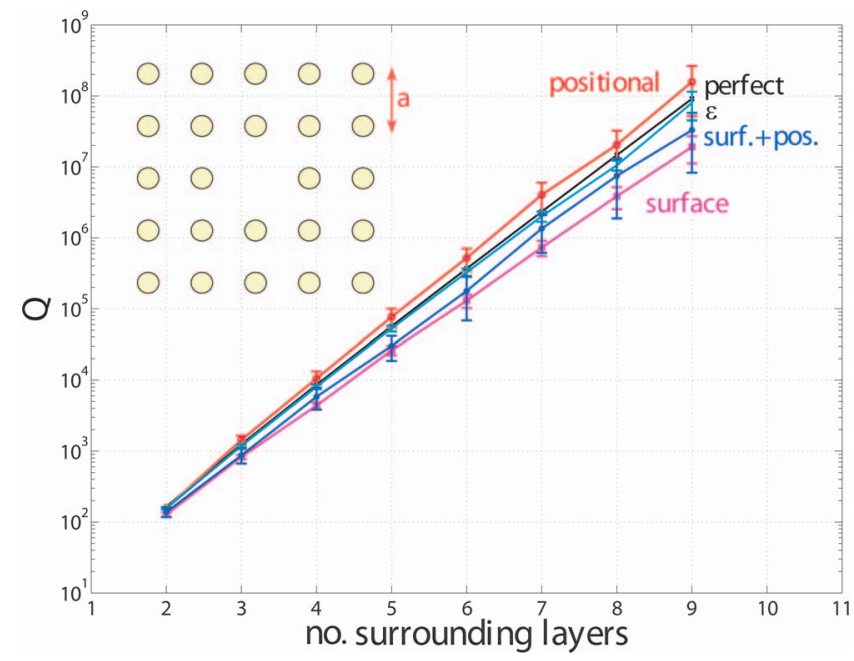

Fig. 1. $Q$ versus number of layers for a $2 \mathrm{D}$ cavity (the inset shows two layers), for disorder types (descending by $Q$ ): position (red), no disorder (black), $\epsilon$ (cyan), surface + position (blue), and surface (magenta). Error bars show the std. dev. over 15 runs. 


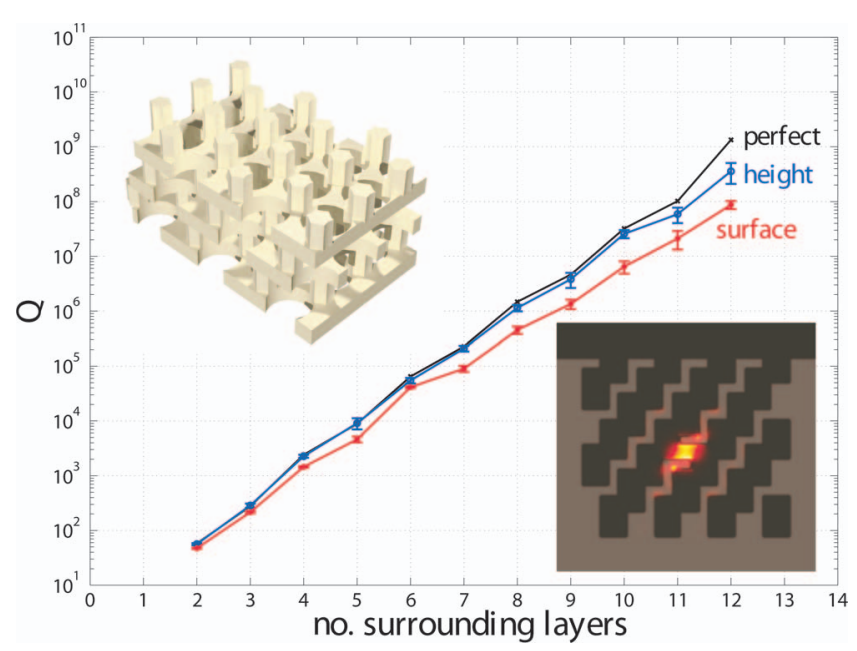

Fig. 2. $Q$ versus the number of layers for cavity in the 3D structure (left inset), for disorder types (descending by $Q$ ): no disorder (black), height (blue), sidewall roughness (red). Right inset: mode $\epsilon|\mathbf{E}|^{2}$ in a vertical cross section (three layers). Error bars show the std. dev. over 15 runs.

constant $a)$ of air cylinders in dielectric $(\epsilon=12)$ oriented along the 111 direction $^{22}$ (radius $0.293 a$, height $0.93 a$ ), forming an alternating stack of rods-in-air and holes-in-dielectric layers with a complete gap (frequencies 0.507-0.628 $2 \pi c / a$ ), shown in the inset of Fig. 2. The 3D structure has an $\epsilon=12$ substrate below and to the sides, and air above with a hole layer topmost, similar to experiment. ${ }^{23}$

In $2 \mathrm{D}$, a cavity is formed by removing a single rod (as in the inset of Fig. 1), giving a single confined mode $^{1}$ with $\omega=0.37792 \pi c / a$ shown in Fig. 3(a). In $3 \mathrm{D}$, we also remove a rod from one of the rods-in-air layers, giving a mode with $\omega=0.56892 \pi c / a$ that resembles the TM mode of the analogous $2 \mathrm{D}$ cavity ${ }^{24}$; a horizontal cross section of this mode is shown in Fig. 3 (a) and a vertical cross section is given in the inset of Fig. 2. Using FDTD with perfectly matched layer absorbing boundaries, ${ }^{13}$ we excite the cavity modes by a Gaussian pulse and extract $\omega$ and $Q$ from the response by a filter diagonalization method. ${ }^{3,25,26} Q$ is defined $^{2,20}$ as the number of optical periods for the energy to decay by $\exp (-2 \pi)$, and modal volume $V$ is computed as $\int \epsilon|\mathbf{E}|^{2} / \max \epsilon|\mathbf{E}|^{2} .{ }^{27}$ For disorder, we compute the mean and standard deviation (std. dev.) for each result over 15 random configurations (except where otherwise specified).

In $2 \mathrm{D}$, with a resolution of 16 pixels/ $a$, we examine four types of random disorder, all with unrealistically large magnitudes: surface [Fig. 3(b), described below], position (random rod-center offsets in $[-0.15 a, 0.15 a])$, surface + position [Fig. 3(c)], and rod $\epsilon$ variations within $[-15 \%, 15 \%]$. For surface disorder, 20 cylinders of random radius in $[0,0.0625 a]$ are added and/or removed from the surface of each rod. Figure 1 shows the resulting $Q$ versus number $n$ of layers around the cavity (e.g., $n=2$ in the inset). Neither the exponential increase of $Q$ (Fig. 1) nor the localized field pattern (Fig. 3) is substantially degraded by even such large disorder. (Indeed, sometimes the disorder happens to improve $Q$, since the perfect structure is not optimized for $Q$.) $\omega$ was $0.37792 \pi c / a$ for the perfect cavity and had a std. dev. of $1 \% / 2 \% / 3 \% / 1 \%$ for surface/position/surf. + pos./ $\epsilon$ disorder, respectively. The modal volume (area) in all cases was approximately $(0.64 \lambda / 2)^{2}$ (std. dev. $\left.<6 \%\right)$.

This insensitivity of confinement to disorder derives from the fact that the bandgap is preserved (only shrunk slightly): disorder introduces states only near the gap edges. We illustrate this by computing the 2D gap as a function of $\epsilon$ disorder strength, plotted in Fig. 4 (using a plane-wave expansion $^{28}$ in a $9 \times 9$ supercell and extracting the largest gap, averaged over 10 runs). The mean FDTD mode $\omega$ and its std. dev. (over 100 runs) is superimposed, corresponding to a well-defined localized mode until the gap shrinks substantially (requiring over $50 \% \epsilon$ disorder). Once the bandgap closes entirely, which is not shown, any observed localized modes have no relation to the original cavity mode, and may correspond instead to Anderson localization.

In $3 \mathrm{D}$, with a resolution of 25 pixels $/ a(\sim 17.7$ pixels per in-plane lattice constant of the $2 \mathrm{D}$ cross sections), we examine two types of random disorder: height disorder through variations $[-5 \%, 5 \%]$ in the height of the air cylinders $( \pm 26 \%$ or $\pm 42 \%$ variation in the thickness of a rod or hole layer, respectively), and sidewall disorder (adding and/or removing cylinders as in 2D) shown in Fig. 3(b). The resulting $Q$ versus number $n$ of surrounding layers is shown in Fig. 2, and the field pattern for sidewall disorder is in Fig. 3(b). ( $n$ is defined so that the number of nearestneighbor rods surrounding the cavity is $n-1$ in plane
$2 \mathrm{D}$

(a)

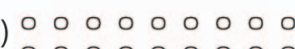
0000000000 0000000000 0000000000 0000000000 0000000000 000000000 0000000000 000000000

(b) 000000000 $\therefore \Leftrightarrow a 000000$ 008000000 000000000 0000 a 000 $\therefore 00000000$ ०० 0 व 0 Q 000000000

(c) 000000000 0 D०००00000 000000000 पर 00000000 - g 0000000 0000000 Cक 0000000 000000000

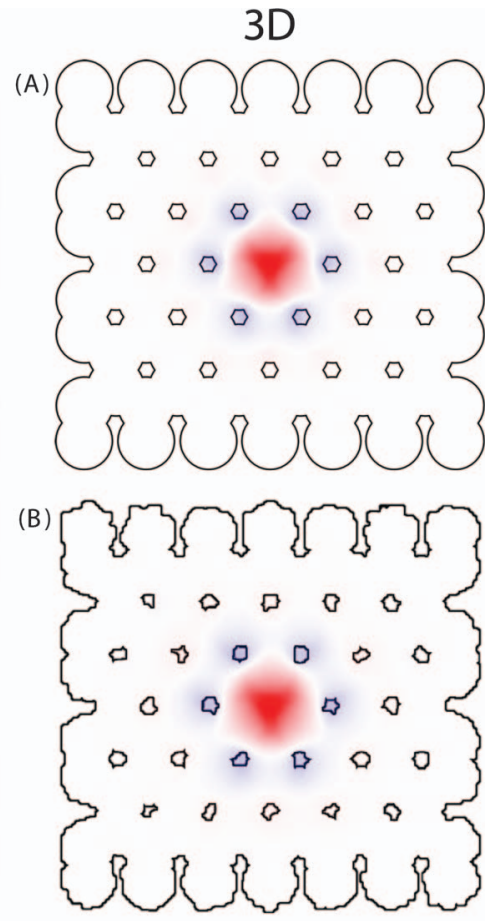

Fig. 3. Cavity mode $E_{z}$ (blue/white/red=positive/zero/ negative) for a $2 \mathrm{D}$ structure with (a) no disorder, (b) surface disorder, and (c) surface+ position disorder, and for a (horizontal) cross section of a $3 \mathrm{D}$ structure with (A) no disorder and $(\mathrm{B})$ sidewall disorder. 


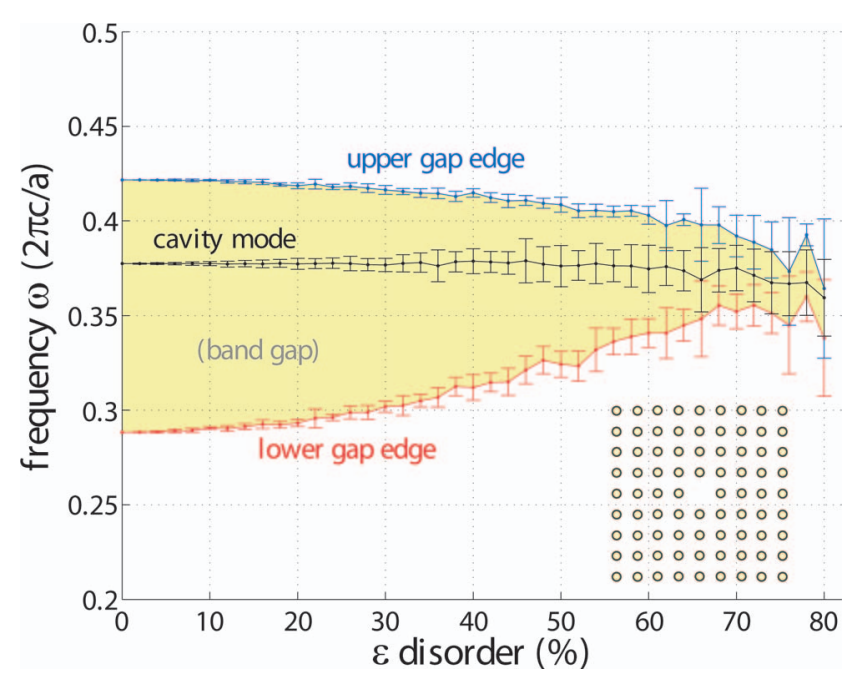

Fig. 4. 2D band gap and cavity mode frequencies versus strength of $\epsilon$ disorder (maximum percent variation of rod $\epsilon)$. Error bars show the std. dev. for 10/100 runs for gap/ cavity frequencies. The inset shows cavity structure (four layers).

and $n$ vertically; the field plots show $n=3$. The total number of vertical periods both above and below is $\sim 2 n / 3$, where a vertical period comprises three layers of holes.) Again $Q$ is only slightly affected by disorder, the modal volume is roughly fixed at $(0.68 \lambda / 2)^{3}$, and frequency $\omega=0.56892 \pi c / a$ has a std. dev. of $0.3 \% / 2 \%$ for the height/sidewall disorder. (Here the sidewall disorder also causes a $3.6 \%$ systematic $\omega$ shift, because addition and removal of cylinders does not have a symmetric first-order effect in $3 \mathrm{D}{ }^{29}$ )

We believe that this robust confinement is a general feature of complete-gap systems for any sufficiently bounded disorder, not limited to the examples here.

This work was supported in part by the Materials Research Science and Engineering Center program of the National Science Foundation under Award DMR9400334, and also by a grant from the Lord Foundation. A Rodriguez's e-mail address is alexrod7 $@$ mit.edu.

\section{References}

1. J. D. Joannopoulos, R. D. Meade, and J. N. Winn, Photonic Crystals: Molding the Flow of Light (Princeton U. Press, 1995).

2. B. E. A. Saleh and M. C. Teich, Fundamentals of Photonics (Wiley, 1991).
3. S. G. Johnson and J. D. Joannopoulos, Photonic Crystals: The Road from Theory to Practice (Kluwer, 2002).

4. M. Lončar, T. Yoshie, A. Scherer, P. Gogna, and Y. Qiu, Appl. Phys. Lett. 81, 2680 (2002).

5. H. Y. Ryu and M. Notomi, Opt. Lett. 28, 2390 (2003).

6. B.-S. Song, S. Noda, T. Asano, and Y. Akahane, Nat. Mater. 4, 207 (2005).

7. M. R. Watts, S. G. Johnson, H. A. Haus, and J. D. Joannopoulos, Opt. Lett. 27, 1785 (2002).

8. S. Fan, P. R. Villeneuve, and J. D. Joannopoulos, J. Appl. Phys. 78, 1415 (1995).

9. H. Li, B. Cheng, and D. Zhang, Phys. Rev. B 56, 10734 (1997).

10. M. A. Kaliteevski, J. Manzanares Martines, D. Cassagne, and J. P. Albert, Phys. Status Solidi A 195, 612 (2003).

11. W. R. Frei and H. T. Johnson, Phys. Rev. B 70, 165116 (2004)

12. A. Yamilov and H. Cao, Phys. Rev. A 69, 031803(R) (2004)

13. A. Taflove and S. C. Hagness, Computational Electrodynamics: The Finite-Difference Time-Domain Method (Artech, 2000).

14. D. M. Pustai, A. Sharkawy, S. Shi, and D. W. Prather, Appl. Opt. 41, 5574 (2002).

15. N. C. Panoiu, M. Bahl, and R. M. Osgood, Opt. Express 12, 1605 (2004), http://www.opticsexpress.org

16. C.-W. Wong, P. T. Rakich, S. G. Johnson, M. Qi, H. I. Smith, Y. Jeon, G. Barbastathis, S.-G. Kim, E. P. Ippen, and L. C. Kimerling, Appl. Phys. Lett. 84, 1242 (2004).

17. T. Asano, W. Kunishi, M. Nakamura, B. S. Song, and S. Noda, Electron. Lett. 41, 37 (2005).

18. T. Tiedje, E. Yablonovitch, G. D. Cody, and B. G. Brooks, IEEE Trans. Electron Devices 31, 711 (1984).

19. M. Soljačić, M. Ibanescu, S. G. Johnson, Y. Fink, and J. D. Joannopoulos, Phys. Rev. E 66, 055601(R) (2002).

20. H. A. Haus, Waves and Fields in Optoelectronics (Prentice-Hall, 1984), Chap. 7.

21. D. W. Vernooy, V. S. Ilchenko, H. Mabuchi, E. W. Streed, and H. J. Kimble, Opt. Lett. 23, 247 (1998).

22. S. G. Johnson and J. D. Joannopoulos, Appl. Phys. Lett. 77, 3490 (2000).

23. M. Qi, E. Lidorikis, P. T. Rakich, S. G. Johnson, J. D. Joannopoulos, E. P. Ippen, and H. I. Smith, Nature 429, 538 (2004).

24. M. L. Povinelli, S. G. Johnson, S. Fan, and J. D. Joannopoulos, Phys. Rev. B 64, 075313 (2001).

25. V. A. Mandelshtam and H. S. Taylor, J. Chem. Phys. 107, 6756 (1997); see also the erratum (Ref. 26).

26. V. A. Mandelshtam and H. S. Taylor, J. Chem. Phys. 109, 4128 (1998).

27. E. M. Purcell, Phys. Rev. 69, 681 (1946).

28. S. G. Johnson and J. D. Joannopoulos, Opt. Express 8, 173 (2001), http://www.opticsexpress.org

29. S. G. Johnson, M. L. Povinelli, M. Soljačić, A. Karalis, S. Jacobs, and J. D. Joannopoulos, Appl. Phys. B 81, 283 (2005). 